\title{
Preoperative planning in paediatric liver tumour surgery - a literature review
}

\author{
Dominika Kościuszko , Agnieszka Niemirycz-Makurat ${ }^{2}$, Stefan Anzelewicz ${ }^{1}$, Andrzej Gołębiewski ${ }^{1}$, Piotr \\ Czauderna ${ }^{1}$ \\ 'Department of Surgery and Urology for Children and Adolescents, Faculty of Medicine, Medical University of Gdansk, Gdansk \\ 80-292, Poland. \\ ${ }^{2}$ Department of Radiology Affidea, M. Kopernik Hospital, Copernicus Medical Company Ltd., Gdansk 80-803, Poland.
}

Correspondence to: Dr. Stefan Anzelewicz, Department of Surgery and Urology for Children and adolescents, Faculty of Medicine, Medical University of Gdansk, Nowe Ogrody street 1-6, Gdansk 80-292, Poland.

E-mail: stefan.anzelewicz@gumed.edu.pl How to cite this article: Kościuszko D, Niemirycz-Makurat A, Anzelewicz S, Gołębiewski A, Czauderna P. Preoperative planning
in paediatric liver tumour surgery - a literature review. Hepatoma Res 2021;7:51. https://dx.doi.org/10.20517/2394-5079.2021.17

Received: 12 Feb 2021 First Decision: 15 Apr 2021 Revised: 30 Apr 2021 Accepted: 26 Apr 2021 First online: 6 Jun 2021

Academic Editor: Guang-Wen Cao Copy Editor: Yue-Yue Zhang Production Editor: Yue-Yue Zhang

\begin{abstract}
Radiological assessment is evolving rapidly, including in paediatric liver tumour surgery. There are advancements in the fields of conventional radiology, 3D imaging and preoperative planning. This article presents the current research in paediatric liver imaging for liver tumour surgery and shows the results of a systematic search in computer-aided liver surgery in children. Sixteen original papers were found. We summarise the progress made and offer the directions in which further research could go. Computer-assisted surgery is a promising field for research and clinical application.
\end{abstract}

Keywords: Paediatric liver tumours, computer-assisted surgery, preoperative planning, paediatric hepatectomy, segmentation

\section{INTRODUCTION}

In 1998, Marescaux et al. ${ }^{[1]}$ described an astounding attempt to apply virtual reality to liver surgery. The authors concluded that surgical planning, training and teaching of liver surgery will be possible using virtual reality concepts. This work has had a significant impact on the development of many new medical technologies. Above all, it has influenced the imagination of surgeons, engineers and computer scientists 
who are in pursuit of change in medical practice. According to the Web of Science (accessed February 2021), the paper has been cited 184 times. However, Marescaux and his team were not the only representatives of this avant-garde. Many others wanted to become a part of the surgical innovation movement, too.

Since that time, information technology, augmented reality, virtual reality, 3D printing and other similar technologies have changed medicine $e^{[2]}$. Many ideas have already been implemented, while others are being rapidly developed. Surgical innovation has always been fundamental to surgical progress ${ }^{[3]}$, and it seems even more apparent at present.

The practice of surgery is changing, and the technological impact can be clearly seen during surgical teaching and learning, as noted by Langridge et al. ${ }^{[4]}$. Additive manufacturing has become a fascinating research topic in anatomy education, surgical training and preoperative planning. Even though this research field is still in its infancy from the point of view of clinical outcomes, technology's impact will probably change the surgical landscape very soon. Technology is becoming cheaper and hence readily available. For instance, Witowski et al ${ }^{[5]}$ proposed in 2017 a cost-effective liver model printed using fused deposition modelling technology. The costs of printing are less than $\$ 150$. Witowski was still a medical student at the time he published these findings.

In 2011, a new concept of so-called precision medicine emerged and was published by the National Research Council of the United States ${ }^{[6]}$. In this model, clinical treatment decisions and other practices are matched to a specific patient or a particular subgroup of patients. The treatment is customised to the patient, and the surgery is tailored to the patient as well. However, the idea of precision liver surgery has been around for a bit longer. Dong et al. ${ }^{[7]}$ made the scientific community aware of the need for a paradigm shift in liver surgery for the first time in 2006 . He called this idea "precision liver resection" ${ }^{[8]}$, but it later evolved to the term "precision liver surgery"[?].

The COVID-19 pandemic has further increased the speed of changes and implementation of telemedicine and other digital technologies. Thus, we can observe more adoption and translation of technology into healthcare.

Inevitably, the old "see one, do one, teach one" paradigm has been shifting ${ }^{[10,11]}$. Now, it seems to be the 4 Ps: plan, prepare, perform and perfect.

This systematic review aims to present studies on computer-aided surgical methods used in paediatric liver surgery, discuss the scientific methods used and provide new perspectives on preoperative planning. This review also aims to discuss progress of the radiological assessment. This paper is organised according to the following sections: plan (radiological assessment), prepare and perform (systematic review) and perfect (discussion).

\section{METHODS}

A comprehensive, focused literature search was conducted and performed according to the Preferred Reporting Items for Systematic Reviews and Meta-Analyses Guidelines ${ }^{[12]}$.

\section{Data sources}

We performed a systematic search using our search strategy in MEDLINE ${ }^{\bullet}$, PubMed $^{\oplus}$, EMBASE and Ovid scientific electronic databases. The keywords were selected based on a recently published mapping of 
research on surgery with mixed reality ${ }^{[13]}$ and crucial articles to create a broader search. The search strategy is described in Table 1. It was conducted on 25 January 2021, and the initial results were screened by three reviewers to identify relevant studies.

\section{Inclusion and exclusion criteria}

Original studies concerning liver surgery in children, preferentially hepatic tumour surgery, were included. In addition, studies concerning virtual reality, augmented reality, mixed reality and 3D printing were included. We excluded articles expressing individual opinions (letters or commentaries), reviews and studies concerning adults, bioengineering and non-medical publications. Studies that did not mention patient age were also excluded.

\section{Data extraction and analysis}

Extracted information included: type of imaging studies performed, software or service used, number of patients, 3D imaging modality, measured outcome variables, the main results and the advantages and disadvantages of different methods.

\section{PLAN (RADIOLOGICAL ASSESSMENT)}

Imaging is the critical components of precision medicine ${ }^{[14]}$ and precision liver surgery. The surgeon expects exact, relevant information from the radiologist. Advances in cross-sectional imaging allow better treatment efficacy and patient safety ${ }^{[15]}$.

The aim of liver tumour imaging is to answer the following questions: is the tumour present, is it malignant or benign and, most importantly, whether complete surgical resection is plausible. To answer them, one should choose the best imaging modality.

The clinician has three main methods of cross-sectional liver evaluation available: ultrasound (US), computed tomography (CT) and magnetic resonance imaging (MRI).

Due to the wide accessibility and patients' small size, US is the primary diagnostic tool for assessing liver tumours in the paediatric population. One can describe the lesion's size, character (solid vs. cystic) and vascularity during ultrasound examination. This assessment is quick and relatively inexpensive. It may be difficult to establish the lesion's extent due to the small field of view, especially when the tumour is large. The inspection for local and distant metastases may be uncertain as well.

Radiation-free imaging, similar to US, is favourable for children as they are much more susceptible to ionising radiation than adults and they are prone to developing radiation-associated side effects, including cancer ${ }^{[16]}$.

Doppler (colour, power, pulsed, etc.) mode can be used to identify the presence or absence of blood flow in a mass, lesion or tumour thrombus in the portal vessels. The characteristics of doppler signal may suggest the organ of origin ${ }^{[17]}$.

Somewhat new, but supported with multiple publications (unfortunately, mainly concerning adults and only scarcely paediatric population), contrast-enhanced ultrasound (CEUS) is emerging as a new liver tumour evaluation method (even though it has been known for over 20 years). 
Table 1. Details of the query search strings used in this systematic review

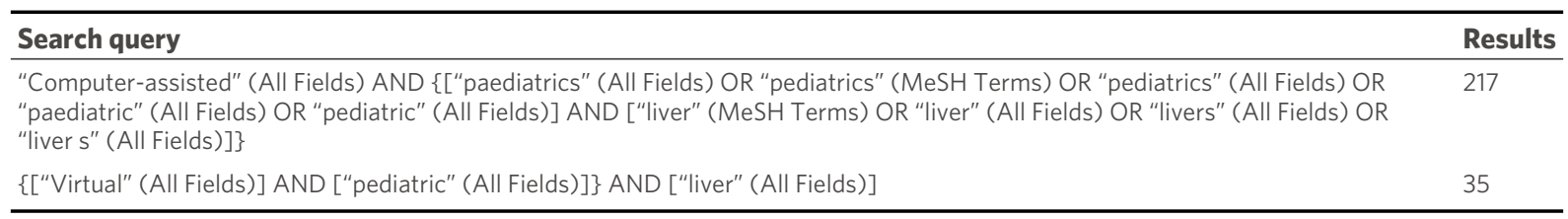

Duplicate articles were removed from the results. The search was performed on 25 January 2021.

Inspired by the contrast agents used in other imaging modalities, ultrasound contrast agents (UCAs) are used to enhance the US's resolution in order to more easily assess the lesion's character. Because of their physical size (1-10 $\mu \mathrm{m}$, i.e., equal to or smaller than red blood cells), UCAs act as blood pool agents and depict both the microvasculature and the macrovasculature ${ }^{[18]}$.

CEUS has been approved globally for liver imaging, most recently in the USA ${ }^{[19]}$. The detection rate of CEUS is comparable to the results of contrast-enhanced magnetic resonance imaging and contrastenhanced computed tomography $(\mathrm{CECT})^{[20]}$. Moreover, UCAs have been proven to be safer than MRI contrast agents (the risk of an anaphylactic reaction is 1:10,000, with few adverse events, and no serious adverse events have been reported $)^{[21,22]}$. The European Federation of Societies for Ultrasound in Medicine and Biology in 2017 stated that UCAs have a good safety profile in adults, and the data in children suggest they are as safe for use in the paediatric population ${ }^{[23]}$. UCAs are also cheaper than MRI or CT.

Examples of hepatic lesions that can be evaluated using CEUS are haemangiomas, telangiectasias and regenerative nodules. They have a characteristic enhancement pattern similar to the one seen on $\mathrm{MRI}^{[24]}$.

More recent publications show that CEUS might be a valuable tool for preoperative assessment of the tumour, as 3D-CEUS volume calculations provided consistent measurements across different readers with almost perfect inter-rater agreement ${ }^{[25]}$ and proved to be accurate. Ablation planning based on $3 \mathrm{D}$ US/CEUS-CT/CEUS fusion imaging has proved to be feasible for liver tumours ${ }^{[26]}$.

Unfortunately, even with Doppler US and the addition of CEUS, the lesion's real character is hard to establish based solely on US.

Therefore, high-resolution CECT and MRI have become the imaging modalities of choice for detailed anatomical assessment of primary paediatric liver tumours ${ }^{[27]}$. The main drawback of both CT and MRI examinations is that they both require sedation in children under 5 years old (and sometimes in older children).

A single portal venous phase CT is generally sufficient when a CT is performed in a paediatric patient to assess a hepatic mass lesion-the lesion's size, vascularity and anatomical position can all be evaluated using solely this phase. The other phases (non-contrast, arterial and delayed) add little diagnostic information ${ }^{[28]}$.

Other risks related to CT (apart from radiation) include the need to use oral and intravenous contrast agents because paediatric patients have little perivisceral fat, making anatomic organs delineation difficult in many $\operatorname{cases}^{[29]}$. 
Simultaneously, modern scanners produce high-quality isovolumetric images that can be reconstructed in multiple imaging planes ${ }^{[25]}$.

Up to date, MRI seems to be the best imaging modality in liver lesions assessment. Several publications have shown that MRI is superior for lesion characterisation of liver pathology when compared to CT. It is only moderately better for lesion detection but contributes clinically crucial additional information in a sizeable number of patients ${ }^{[30-32]}$.

MRI has the advantages of providing excellent soft tissue contrast without exposing the patient to ionising radiation. Advances in both MRI hardware and software technology have significantly contributed to the detection, characterisation and staging of hepatic neoplasms ${ }^{[33]}$.

MRI in paediatric patients can be performed with either 3 or $1.5 \mathrm{~T}$ systems $\mathrm{s}^{[34]}$. Imaging at $3 \mathrm{~T}$ has a better signal-to-noise ratio and spatial resolution than $1.5 \mathrm{~T}$ imaging when combined with acceleration techniques such as parallel imaging and compressed sensing ${ }^{[35]}$, which makes it preferable in younger children ${ }^{[3,37]}$.

Although new, but with proven advantages, hepatobiliary agents have been used to evaluate the biliary system's anatomy, function and pathology ${ }^{[38]}$.

There are three FDA-approved MR contrast agents with differing degrees of hepatocyte uptake and biliary excretion. Gadoxetic acid is discussed in more detail in this paper. It has been marketed in the US since 2008 (approved as Primovist ${ }^{\oplus}$ in Europe since 2004). Almost 50\% of gadoxetic acid is taken up by hepatocytes and excreted into biliary canaliculi ${ }^{[39]}$. Hepatobiliary phase liver imaging shows peak contrast enhancement at $20 \mathrm{~min}$ with a persistent enhancement for more than $2 \mathrm{~h}^{[40]}$. The early enhancement is the main reason this agent is preferred in the paediatric population ${ }^{[41]}$. Gadoxetate is safe in children older than two months ${ }^{[42]}$. The advantages of gadoxetate are improved characterisation of liver lesions, enhanced detection of metastases, better evaluation of the relationship of tumours to the biliary tree (to evaluate the biliary tree for benign disease and for tumours communicating with the biliary tree $\left.{ }^{[43]}\right)$ and increased diagnostic confidence of focal nodular hyperplasia (FNH) differentiation from liver metastases ${ }^{[39]}$. If needed, they can guide the biopsy of more suspicious lesions ${ }^{[44]}$. This last benefit is significant in children who have been treated for a primary tumour, as there is an increased incidence of FNH in those patients ${ }^{[45]}$.

\section{PREPARE AND PERFORM (SYSTEMATIC REVIEW)}

We found 16 relevant articles that contribute to the application of $3 \mathrm{D}$ visualisation in paediatric liver surgery. The papers are summarised in Tables 2 and 3. Table 4 summarises the outcome measures used in the reviewed research studies.

Plumley et al ${ }^{[46]}$ were the first to describe the role of helical (spiral) CT scan, with 3D reconstruction, in the preoperative assessment of solid paediatric tumours. From the nine described cases, one patient was a child with hepatoblastoma. The surgical treatment was feasible after performing the spiral-CT scan and $3 \mathrm{D}$ reconstruction, even though initially the tumour seemed unresectable. The authors believed that the excellent resolution of vascular structures and the ability to evaluate the tumour's $3 \mathrm{D}$ relationship to these structures in multiple projections allow preoperative surgical strategies that result in safe and complete surgical resection of tumours. 
Table 2. A concise description of relevant articles, sorted by year of publication (ascending) and the last name of the first author

\begin{tabular}{|c|c|c|c|c|}
\hline Ref. & $\begin{array}{l}\text { Year of } \\
\text { publication }\end{array}$ & Study type & Study description & $\begin{array}{l}\text { Sample } \\
\text { size }\end{array}$ \\
\hline Plumley et al. ${ }^{[46]}$ & 1995 & Case report & $\begin{array}{l}\text { Assessment of tumour size and location in relation to } \\
\text { vital structures }\end{array}$ & $1(9)^{\star}$ \\
\hline Fuchs et al. ${ }^{[47]}$ & 2005 & Evaluation study & Preoperative 3D visualisation and virtual resection & $4(11)^{\star}$ \\
\hline Dong et al. ${ }^{[48]}$ & 2007 & Validation study & $\begin{array}{l}\text { Comparison between 3D preoperative reconstruction vs. } \\
\text { conventional CT }\end{array}$ & 18 \\
\hline Fuchs et al. ${ }^{[49]}$ & 2010 & Evaluation study & $\begin{array}{l}\text { Description of experience with the use of software } \\
\text { assistants }\end{array}$ & 12 \\
\hline Souzaki et al. ${ }^{[50]}$ & 2013 & Case report & $\begin{array}{l}\text { Description of a novel augmented reality navigation } \\
\text { system }\end{array}$ & $1(6)^{\star}$ \\
\hline Souzaki et al. ${ }^{[51]}$ & 2015 & Case report & 3D-printed liver model based on preoperative CT scan & 1 \\
\hline Su et al. ${ }^{[52]}$ & 2015 & Evaluation study & $\begin{array}{l}\text { Experience with a computer-assisted surgery system in } \\
\text { liver resections }\end{array}$ & 21 \\
\hline Soejima et al. ${ }^{[53]}$ & 2016 & Case report & $\begin{array}{l}\text { Description of a 3D-printed model of a liver graft and the } \\
\text { recipient's body }\end{array}$ & 1 \\
\hline Su et al. ${ }^{[54]}$ & 2016 & ComparativeRetrospective & $\begin{array}{l}\text { Comparison between 3D preoperative reconstruction vs. } \\
\text { conventional CT }\end{array}$ & 26 \\
\hline Warmann et al. ${ }^{[55]}$ & 2016 & Evaluation study & $\begin{array}{l}\text { Description of experience with the use of software } \\
\text { assistants }\end{array}$ & 24 \\
\hline Zhang et al. ${ }^{[57]}$ & 2016 & Validation study & $\begin{array}{l}\text { Comparison between 3D preoperative reconstruction vs. } \\
\text { conventional CT }\end{array}$ & 21 \\
\hline Janek et al. ${ }^{[58]}$ & 2017 & Evaluation study & $\begin{array}{l}\text { Experience with a computer-assisted surgery system in } \\
\text { liver resections }\end{array}$ & 5 \\
\hline Zhao et al. ${ }^{[59]}$ & 2017 & Evaluation study & $\begin{array}{l}\text { Evaluation of a computer-assisted surgery system in } \\
\text { preoperative surgical planning and intraoperative } \\
\text { navigation }\end{array}$ & 5 \\
\hline Wang et al. ${ }^{[60]}$ & 2019 & Validation study & $\begin{array}{l}\text { Application of 3D-printed liver models in paediatric } \\
\text { living-donor liver transplant }\end{array}$ & 30 \\
\hline Esaki et al. ${ }^{[61]}$ & 2020 & Validation study & $\begin{array}{l}\text { Segmentation and volumetry of a liver graft using U-Net } \\
\text { convolutional neural network }\end{array}$ & $100 / 20^{\#}$ \\
\hline Ishii et al. ${ }^{[63]}$ & 2020 & Case report & $\begin{array}{l}\text { Experience with patient-specific 3D-printed liver model } \\
\text { based on preoperative } \mathrm{CT}\end{array}$ & 1 \\
\hline
\end{tabular}

"The number refers to patients with liver tumours; the number in parentheses refers to the number of all patients. "100 patients were used for learning and 20 patients for validation. Note that not all patients were children in this study.

Fuchs et al ${ }^{[47]}$ published in 2005 one of the first reports of using 3D CT reconstructions in paediatric liver surgery. The authors found an excellent correlation between $3 \mathrm{D}$ reconstructions and the findings during surgery. Tumour resections could be planned more precisely. Especially the colour-coded determination of risk margins seemed to be useful. The authors used MeViS services (Fraunhofer Institute for Digital Medicine). Within this service, surgeons receive images of the individual cases comparable to a personal anatomical textbook together with an automatic risk analysis. Through the analysis of separate liver territories (according to the portal venous drainage), the authors also noted variations of the Couinaud's classification. Individual differences in the segmental borders within the liver were found. The authors also stated that anatomic variants could be demonstrated and evaluated reliably.

Dong et al.$^{[48]}$ in 2007 analysed large tumours (> 30\% of hepatic mass) or tumours at the hepatic hilum. Three-dimensional CT imaging provided high-quality images of the tumours and their borders and the tumour location relative to vital hepatic blood vessels (before this technique, angiography was used, which had many disadvantages). As the surgeons identify hepatic blood vessels before surgery, they can reduce the risk of massive haemorrhage. The authors concluded that $3 \mathrm{D}$ reconstruction offers a more accurate method than traditional imaging techniques. It could help the surgeon identify the tumour borders precisely and devise a comparatively safe surgical strategy. 
Table 3. A summary of the types of preoperative imaging studies used, as well as the software and types of technology implemented in the study

\begin{tabular}{|c|c|c|c|}
\hline Ref. & Preoperativestudy used & Software used & What was performed \\
\hline Plumley et al. ${ }^{[46]}$ & CT & No data & $3 \mathrm{D}$ reconstruction \\
\hline Fuchs et al. ${ }^{[4]}$ & $\mathrm{CT}$ & MeVis service & $3 \mathrm{D}$ reconstruction \\
\hline Dong et al. ${ }^{[48]}$ & $\mathrm{CT}$ & 4D Recon Card Wizard (Siemens) & $3 \mathrm{D}$ reconstruction \\
\hline Fuchs et al. ${ }^{[49]}$ & $\mathrm{CT}$ & MeVis service & $3 \mathrm{D}$ reconstruction \\
\hline Souzaki et al. ${ }^{[50]}$ & $\mathrm{CT} / \mathrm{MRI}$ & Virtual Place 300, AZE Co (Now AZE Virtual Place, Cannon) & Augmented reality \\
\hline Souzaki et al. ${ }^{[51]}$ & $\mathrm{CT}$ & ZedView, 3D Doctor, FreeForm, CATIA & 3D printing \\
\hline Su et al. ${ }^{[52]}$ & $\mathrm{CT}$ & Higemi & $3 \mathrm{D}$ reconstruction \\
\hline Soejima et al. ${ }^{[53]}$ & $\mathrm{CT}$ & Free Form, K's Design Lab & 3D printing \\
\hline Su et al. ${ }^{[54]}$ & $\mathrm{CT}$ & Hisense CAS system & $3 \mathrm{D}$ reconstruction \\
\hline Warmann et al..$^{[55]}$ & $\mathrm{CT} / \mathrm{MRI}$ & MeVis service & $3 \mathrm{D}$ reconstruction \\
\hline Zhang et al. ${ }^{[57]}$ & $\mathrm{CT}$ & Hisense CAS system & $3 \mathrm{D}$ reconstruction \\
\hline Janek et al. ${ }^{[58]}$ & $\mathrm{CT}$ & MeVis service & $3 \mathrm{D}$ reconstruction \\
\hline Zhao et al. $^{[59]}$ & $\mathrm{CT}$ & Hisense CAS system & $3 \mathrm{D}$ reconstruction \\
\hline Wang et al. ${ }^{[60]}$ & $\mathrm{CT} / \mathrm{MRI}$ & IQQALiver; EDDA Technology & 3D printing \\
\hline Esaki et al. ${ }^{[61]}$ & $\mathrm{CT}$ & U-Net and three-dimensional (3D) workstations (3DWS) & Deep learning \\
\hline Ishii et al. ${ }^{[63]}$ & СТ & Synapse Vincent (Fujifilm) & 3D printing \\
\hline
\end{tabular}

Table 4. Outcome measures

\begin{tabular}{|c|c|}
\hline Ref. & Measured outcomes \\
\hline Plumley et al. ${ }^{[46]}$ & Recurrences, kidney function, changing the operation plan \\
\hline Fuchs et al. ${ }^{[4]}$ & The margin of resection, intraoperative complications, portal venous thrombosis \\
\hline Dong et al. ${ }^{[48]}$ & Operation time, blood loss, mortality, surgeons subjective opinion \\
\hline Fuchs et al. ${ }^{[49]}$ & The margin of resection, intraoperative complications, portal venous thrombosis \\
\hline Souzaki et al. ${ }^{[50]}$ & Operation time, reoperations, mortality, intra- and postoperative complications \\
\hline Souzaki et al. ${ }^{[51]}$ & Recurrence, the margin of resection \\
\hline Su et al. ${ }^{[52]}$ & Operation time, blood loss, blood transfusion, mortality \\
\hline Soejima et al. ${ }^{[53]}$ & Operation time, blood loss, blood transfusion, mortality, postoperative complications \\
\hline Su et al. ${ }^{[54]}$ & Operation time, blood loss, blood transfusion, mortality, postoperative complications, recurrence, RSLVR \\
\hline Warmann et al. ${ }^{[55]}$ & Mortality, the margin of resection, changing operation plan \\
\hline Zhang et al. ${ }^{[57]}$ & Operation time, blood loss, intra- and postoperative complications, postoperative stay, the margin of resection, recurrence \\
\hline Janek et al. ${ }^{[58]}$ & Blood loss, blood transfusion, recurrence, bilirubin levels, the volume of post-resection liver residue \\
\hline Zhao et al. ${ }^{[59]}$ & Operation time, blood loss, blood transfusion \\
\hline Wang et al. ${ }^{[60]}$ & Operation time, blood loss, mortality, intra- and postoperative complications, inpatient stay, cost \\
\hline Esaki et al. ${ }^{[61]}$ & Liver and spleen volume measurement \\
\hline Ishii et al. ${ }^{[63]}$ & Operation time, blood loss, intraoperative complications \\
\hline
\end{tabular}

In 2010, Fuchs et al. ${ }^{[49]}$ proposed an approach to the planning of surgical treatment of liver vascular malformations in children. This approach incorporated MeVis LiverAnalyzer and MeVis LiverExplorer (Mevis, Bremen, Germany). Twelve children were diagnosed with hepatic vascular malformations. The patients were assessed for Rex shunt procedure and other interventions. Liver segmentations were performed using the livewire segmentation technique. This semiautomatic technique needs user input to set anchors around the region of interest. The smallest "cost path" is then drawn from the anchor to the pixel where the pointer is. The path is changed whenever the user moves the pointer. 
The authors found that virtual reconstructions provided information that was not obtained through conventional imaging techniques. They qualitatively explored the possibilities for the use of 3D imaging. The only measurable result provided is the number of procedures performed: seven patients received interventions (out of twelve). The authors also found that combining reconstructions from different CT phases on one $3 \mathrm{D}$ image provided further useful information. The authors had difficulty distinguishing between portal branches and collaterals, and reduced contrast in the post-thrombotic vessels did not allow proper identification.

In 2013, Souzaki et al. ${ }^{[50]}$ presented an augmented reality navigation system for endoscopic and open surgery. In their case series, one patient had been diagnosed with hepatoblastoma. Initial CT and MRI studies were performed with multimodality markers (Chiyoda Technol, Tokyo, Japan) fixed to the patient's body surface. 3D images were reconstructed using 3D viewer software (Virtual Place 300, AZE Co Ltd., Tokyo, Japan). Each multimodality marker's coordinates were obtained with an optical tracking device (Polaris, Nrthern Digital Inc., Ontario, Canada). A video camera registering the surgical image had a rotary encoder to measure the rotation parameters. Liver borders were detected by the system, and preoperative $3 \mathrm{D}$ images were superimposed onto video camera live images on the screen. The authors' most significant difficulty was concerning motion and deformation of the intraoperative images of the liver. Respiratory movement and deformation caused by pressure are essential factors that create differences between the images and reality. Due to intraoperative organ movement, it was impossible to follow the intraoperative organ deformations. The authors also found that the $3 \mathrm{D}$ image projected onto $2 \mathrm{D}$ monitors produced dissatisfying results.

Souzaki et al ${ }^{[51]}$ published in 2015 a case report of using a 3D-printed liver model based on preoperative CT for a PRETEXT IV hepatoblastoma case, located at the porta hepatis after the neoadjuvant chemotherapy. The model was created in a 3D workstation with ZedView, 3D Doctor, FreeForm (analytical software) and CATIA, a three-dimensional interactive application. The model was printed on an Objet 500 connex 3 printer (Stratasys, USA) with the tumour, hepatic veins and portal veins seen through a translucent body. The tumour was resected completely. The surgical margins were negative, and the patient was discharged home. No recurrence was detected for a year. The authors found that the object can be sterilised and held in the surgeon's hand during surgery. Compared with $3 \mathrm{D}$ images, the authors found that the $3 \mathrm{D}$ model after fabrication cannot be changed and manipulated during surgery.

Su et al.$^{[52]}$ published, also in 2015, a case series of 21 paediatric patients with complex liver tumours. The authors used Hisense Gemini 3-dimensional Medical Imaging Reconstruction (HIGEMI) and a computerassisted surgery (CAS) system. The images were segmented using the seeding technique. They segmented the liver, tumour, hepatic arteries, portal vein, hepatic veins and inferior vena cava. The authors recorded histopathological reports, duration of surgery, intraoperative blood loss, transfusions and complications. One patient died. This is the only paper that mentions the HIGEMI system.

Soejima et al.$^{[53]}$ described the case of an 11-month-old girl with biliary atresia after Kasai surgery who had to undergo a liver transplant. The transplant turned out to be large-of-size and impossible to perform from the CT image's initial calculations. For a more detailed analysis of this area's anatomy, the researchers decided to make 3D models of the donor and recipient liver. ZedView (Data Design, Nagoya, Japan) software was used to prepare the models. Thanks to those models, the resection line was changed to a more optimal one, and the operation was planned. The surgery proceeded without intraoperative complications; only slight blood loss was noted, and transfusion was not needed. Unfortunately, the postoperative course was complicated by infection. The patient died due to an intracranial haemorrhage 135 days after the 
transplant (while the graft was still functioning).

Su et al. ${ }^{[54]}$ described a study conducted on 26 children aged 22 days to 36 months with giant liver tumours. The Hisense CAS system was used in the reconstruction group and CT workstation examination in the control group. Based on the reconstruction, the procedure was simulated. The $3 \mathrm{D}$ images were also displayed in the operating room during the operation. It was concluded that the $3 \mathrm{D}$ images can precisely locate the tumour and its vascularisation. Eight patients received blood transfusions, and the mean operative time was shorter in the study group. The mean intraoperative blood loss was lower in the study group. Both differences were statistically significant.

Warmann et al. ${ }^{[55]}$ designed a study that aimed at defining the role of computer-aided surgical planning in children with advanced hepatic tumours, i.e., those affecting three or four sectors of the liver. The primary outcome was the possibility to avoid liver transplantation. The authors used the MEVIS service. Surgical planning is offered by MEVIS as a commercial product, and results are embedded in a 3D interactive PDF file ${ }^{[56]}$. Sixty-three patients were evaluated, but only 24 had computer-aided surgical planning performed. Most of the patients had a CT-scan, but, interestingly, the authors did not see any difference in quality between CT scans and MRI scans. The authors stated that the 14 patients with hepatoblastoma affecting three or four liver sectors post-chemotherapy (POST-TEXT III or IV) were potential candidates for liver transplantation, but, finally, only two patients were qualified for a liver transplant. The authors observed close relationships between computer-aided surgical planning and intraoperative findings. Resection margins were free of tumour cells. The authors found variations and significant differences in segmental distribution between the patients. Variations in segment volume were presented as mean percentage and ranges. They analysed the disease-free survival rates of the hepatoblastoma patients, and the overall survival rate was $90 \%$ after five years.

In 2016, Zhang et al. ${ }^{[57]}$ published a cohort study comparing two groups of children who underwent hepatectomy. Before surgery, all children had CT scans performed. CT images of eleven children were reconstructed using Hisense CAS, and 10 children were operated on without performing $3 \mathrm{D}$ reconstructions of the images. The two study groups were compared. No positive margins, no complications and no recurrences of the disease were noted in both groups. The authors stated statistically shorter surgery duration, less intraoperative bleeding and shorter hospital stay in the reconstruction group.

Another study was published in 2017 by Janek et al ${ }^{[58]}$. They also used MeVis distant services for surgical planning. Their cohort consisted of five children diagnosed with hepatoblastoma. Post-surgical liver volume was $28 \%-70 \%$, and none of the children suffered from post-resection liver failure. Liver volumetry was performed in OsiriX (at the time of diagnosis) and by MeVis (after neoadjuvant chemotherapy). The lowest recorded FRL equalled 28\% and was well tolerated. The authors also used OsiriX for liver volumetry but found the analysis time-consuming. The authors also stressed that risk analysis performed by $\mathrm{MeVis}$ is fundamental, and the $3 \mathrm{D}$ topographic-anatomical visualisation is complete. The analysis by $\mathrm{MeVis}$ also contains suggested resections with calculations of liver volumes. In one of the patients, the authors used Biliskopin $^{\text {tu }}$ for the analysis of the biliary tract. The price of the MeVis analysis is about 700-800 euros, as stated by the authors.

Five children with hepatic mesenchymal hamartomas participated in a study by Zhao et al. ${ }^{[59]}$. The children were 8-24 months old. 3D liver reconstructions were prepared for all children based on CT examinations, including three children using the Hisense CAS software. With this software, a virtual hepatectomy was performed with the calculation of the residual volume. Based on the simulation, the optimal surgical 
technique was selected. The paper presents values such as the time of surgery, bleeding and the need for blood transfusion concerning the tumour's size. There were no postoperative complications in any of the patients. Patients were followed-up for one year - no recurrence occurred. The intraoperative appearance of the liver was consistent with the reconstruction image. Reduced blood loss and shorter surgery time were also found in reconstructive patients. It was also noted that such reconstructions can be useful for learning by students and young surgeons.

Another experience was published by Wang et al. ${ }^{[60]}$ Thirty patients requiring living donor liver transplantation were enrolled in the study. In this study, a preoperative simulation was carried out, and models were created on its basis. The segmentation method was not given-the organ volume was calculated automatically. The median age of recipients was 23 months in the study group and 11 months in the control group. The median age of the donors was 34 and 35 years, respectively. The authors pointed out the disadvantage of $3 \mathrm{D}$ reconstruction displayed on a flat screen. Printed models allow better 3D visualisation. Additionally, thanks to transparent silicone for parenchyma, it is easy to visualise the internal course of the vessels and the tumour's location. Ultimately, no statistically significant difference was found for surgery, blood loss, length of stay, cost or survival. Fewer patients had complications, but the result was also statistically insignificant. The duration of donor surgery was significantly shortened. Follow-up lasted an average of 35 months.

Esaki et al. ${ }^{[61]}$ published a paper that is unlike the other works presented in this review. They proposed a method that uses a convolutional neural network (U-net), developed for biomedical image segmentation at the Computer Science Department of the University of Freiburg ${ }^{[62]}$. U-net is provided as open-source software. The method was used for automatic segmentation of liver graft volume of paediatric liver transplant patients. The accuracy of automatic segmentation was assessed after the network learned using 100 patient datasets. The accuracy was measured using Dice similarity coefficients on 20 patient datasets. The accuracy rate for liver graft was $87.10 \% \pm 4.70 \%$. The authors used a neural network to accelerate the segmentation process. Computed tomography datasets were used for training of the neural network and for verification of its efficacy. The segmentations created by the U-net were compared to those made by an experienced radiology technician and verified by radiologists.

Ishii et al. ${ }^{[63]}$ described a single case of using a 3D-printed model for a 22-month-old girl with biliary atresia, leading to a liver transplant. An additional anatomical difficulty was situs inversus - a rare congenital defect causing all internal organs' vertical inversion. During the model's standard creation, the accuracy was assessed by scanning with an industrial CT system. After sterilisation, the printed model was taken to the operating room to compare its anatomy with the actual patient. The authors pointed out that the perception of the dorsal side of liver anatomy is more straightforward, thanks to the $3 \mathrm{D}$ model that could be easily rotated in the hands. The operation time was $646 \mathrm{~min}$, blood loss was $425 \mathrm{~mL}$, and the operation was completed without any intraoperative complications. The developers noted and quoted the time it takes to create the model: it took about 30-60 min to generate STL files from the original CT data. Then, it usually took about three days to produce 3D liver models from STL files. An additional day was needed for ethylene oxide sterilisation. Accordingly, the entire process takes 4-5 days.

\section{DISCUSSION (PERFECT)}

Complete surgical resection of hepatoblastoma is considered the most crucial step to achieve long-term survival ${ }^{[64]}$. Open surgery is most commonly performed ${ }^{[65]}$, but laparoscopic liver surgery is also a feasible, yet very rarely used, approach ${ }^{[6]]}$. The International Laparoscopic Liver Society members encourage routine use of laparoscopy in local liver resections and left lateral segmentectomies ${ }^{[67]}$. One of the reviewed studies, 
by Souzaki et al..$^{[50]}$, utilised an augmented reality navigation system in laparoscopic surgery for two cases (Wilms' tumour and bronchogenic cyst). In the hepatoblastoma case, the augmented reality navigation system was used for open surgery. The authors' main hindrance was the increasing difference between the AR image and reality with the operated organs' displacement and compression. The system could not follow organ deformations. Marescaux et al. ${ }^{[68]}$ reported the first real-time AR laparoscopic adrenalectomy. That system used manually assisted deformable registration. Haouchine et al. ${ }^{[60]}$ proposed another solution for rigid registration and published a paper the same year as Souzaki et al. ${ }^{[51]}$. Haouchine et al. ${ }^{[60]}$ suggested a real-time, physics-based system able to automatically register deformable organs using stereo endoscopy. It was shown in 2009 that stereoscopic endoscopes can take accurate anatomical measurements ${ }^{[6]]}$.

Because excellent knowledge of the liver's anatomy is crucial for successful resection (or transplantation) and protection of the vascular inflow and outflow to the remaining liver ${ }^{[65]}$, it is believed that $3 \mathrm{D}$ visualisation may help in the surgical treatment of liver tumours.

All of the reviewed papers speak favourably of computer-aided surgery. However, Warmann et al. ${ }^{[55]}$ claimed that, owing to 3D reconstructions, 12 out of 14 hepatoblastoma patients could avoid transplantation. Dong et al. ${ }^{[48]}$ also stated that $3 \mathrm{D}$ surgical planning allowed more comfortable and safer dissection. The authors declared that this technique should be applied especially in large or complex liver tumours.

The critical, post-resection volume of the liver is called the future liver remnant (FLR). This is an essential concept because it was proven that, in the case of FLR $<20 \%$, more than half of patients develop severe postoperative complications, while, for FLRs $>20 \%$, the risk of liver failure is $13 \%{ }^{[70]}$. Vauthey et al. ${ }^{[7]]}$ stated that the minimal safe FLR volume is $25 \%$ for cases of extended right-sided hemihepatectomy. Shoup et al. ${ }^{[72]}$ also found that an FLR volume that is less than $25 \%$ is a significant risk for hepatic dysfunction. There is no consensus regarding the minimal FLR that is safe for liver surgery in children.

Most of the studies used medical imaging datasets obtained from CT studies. Only Souzaki et al..$^{[50]}$, Warmann et al..$^{[55]}$ and Wang et al. ${ }^{[60]}$ reported the use of data from MRI studies. This is because MRI images are less accurate, have a higher signal-to-noise ratio than CT and have lower image contrast differences between tissues. Fuchs et al. ${ }^{[49]}$ noted that the spatial resolution of CT allows for more detailed reconstructions when compared to MRI. In addition, the thickness of the slices is essential for $3 \mathrm{D}$ reconstructions. The problem also lies in inhomogeneous signal intensities. Those obstacles can be avoided through the proper design of an MRI protocol ${ }^{[73]}$. However, good communication between the radiologist, MRI team and the person who wants to use the MRI dataset for 3D reconstruction is necessary.

Liver volumetry is one of the essential computer-aided surgical tools. IT can be performed by surgeons alone. Van der Vorst $^{[74]}$ presented accurate volumetric measurements using Osirix ${ }^{\bullet}$ (Pixmeo, Geneva, Switzerland). Dello et al..$^{[75]}$ compared volumetry done in Osirix ${ }^{\odot}$ and ImageJ by non-radiologists on personal computers and compared the results with radiological software iNtuition ${ }^{\circ}$ (National Institute of Health, USA). The results were accurate. In another paper, Dello et al. ${ }^{[76]}$ presented how to perform liver volumetry in ImageJ. Lodewick et al. ${ }^{[77]}$ compared volumetry in OsiriX ${ }^{\bullet}$ and iNtuition ${ }^{\circledR}$ and also found no differences in measured volumes, but they also stressed that auto-segmentation with iNtuition ${ }^{\bullet}$ is three times faster than manual volumetry in OsiriX ${ }^{\circledR}$. In the reviewed articles, volumetry was performed by radiologists or software engineers. 
The currently used systems rely on software assistants ${ }^{[57]}$ that use specific software packages such as iNtuition ${ }^{\circ}$, Fraunhofer MeVis or Visible Patient Planning ${ }^{\text {twi }}$ (Ircad, France; not mentioned in the reviewed articles). Those software packages or services include medical image analysis with deep learning and machine learning techniques: automated organ segmentation in CT and MR, automatic liver lesion detection and segmentation, automatic vessel segmentation in contrast - enhanced CT, etc. Several opensource neural networks exist that can perform automatic segmentation tasks, such as U-net ${ }^{[78]}$. A complete automated liver segmentation problem is not fully solved yet, although the situation has changed since $2009^{[79]}$ thanks to deep neural networks ${ }^{[80,81]}$. The paper by Ibtehaz et al. ${ }^{[78]}$ is different from the other reviewed articles, as it describes U-net's use in automatic volumetry of liver graft.

Several well-designed review have been published recently on augmented and virtual reality in oncologic liver surgery $y^{[14]}, 3 \mathrm{D}$ printing in liver surgery ${ }^{[82]}, 3 \mathrm{D}$ printing in general medical setting ${ }^{[83]}$, precision hepatectomy ${ }^{[84]}$ and liver segmentation techniques ${ }^{[85]}$. However, we feel that this landscape is incomplete due to the lack of a comprehensive review of recent advances in radiological assessment of the liver focused on preoperative liver surgery planning in the paediatric population. We believe that this focused approach on the topic is essential because it enables a thorough analysis of the achievements in the field. In other words, it allows us to define "where we are".

The reviewed studies have mostly evaluative characteristics or are simply case reports. Only Zhang et al. ${ }^{[57]}$, Su et al.$^{[54]}$ and Wang et al ${ }^{[60]}$ compared the results of patients who had computer-aided surgical planning performed with patients without additional planning. However, there is a high risk of bias in those studies, mainly confirmation bias. Due to the low numbers of patients in the groups, the studies may be underpowered and not show significant results. More prospective, comparative studies with well-defined outcome measures should be performed. It is also not known whether the computer-aided preoperative planning should be used in all cases or only in complex cases.

It is also not known how surgeons perceive different types of $3 \mathrm{D}$ preoperative planning. What is the difference between 3D-printed object, 3D visualisation seen on a computer screen and using 3D glasses or mixed reality glasses? Even subjective opinions of surgeons would be helpful to choose the best tool for further evaluation. The use of these advanced methods during surgery may be problematic because, for now, they need active input from the surgeon (to hold the 3D-printed model or ask someone to hold and move the model for her/him).

Most authors used 3D reconstructions presented on monitors and 3D-printed objects. The application of augmented reality utilising stereo head-mounted displays enabling holographic imaging has not been described yet in paediatric liver tumour surgery. An example of such a device is Microsoft's HoloLens ${ }^{\mathrm{rm}}$. The $3 \mathrm{D}$ models can be manipulated during surgery by the operating surgeon and tracked by hand gestures and voice commands. The use of HoloLens ${ }^{\mathrm{Tm}}$ in adult liver surgery has been described ${ }^{[8]}$, and the results are favourable.

On the other hand, a holographic image still needs to be set appropriately (zoomed-in or out, rotated, etc.). When overlying the operated organ, the holographic image may partly cover the organ and distract the surgeon. In addition, the $3 \mathrm{D}$-printed models and $3 \mathrm{D}$ visualisations presented on the screens may be a source of distraction. It has not been yet resolved whether computer-aided surgery is beneficial only before the operation (preoperative planning) or during the surgery, despite all enthusiasm. This may also be a question of a long learning curve. In addition, there is a possibility that experienced surgeons will be less likely to use novel technologies. However, this technology may also work as a booster of a learning curve in 
hepatobiliary surgery because it seems much more comfortable to grasp the anatomy while using 3D models and visualisations.

Another promising advancement is cinematic rendering (Siemens Healthineers), a new type of 3D imaging with a realistic photo quality visualisation. This novel rendering algorithm simulates the propagation and interaction of light passing through the volumetric images. Elshafei et al ${ }^{[87]}$ compared cinematic rendering with conventional computed tomography imaging in a randomised, two-sequence crossover study. Cinematic rendering allowed for faster and more correct comprehension of the surgical anatomy. A similar conclusion was drawn by Binder et al. ${ }^{[88]}$ who studied cinematic rendering in preclinical anatomy education. The use of cinematic rendering of focal liver masses was described in $2019^{[89]}$.

\section{CONCLUSIONS}

Paediatric liver tumours are rare, and, in some complex cases, the treatment approach has to be made individually. This is one of the instances where $3 \mathrm{D}$ imaging starts to play a role. It is possible that computeraided surgical planning will become routine in all major surgeries. Due to technical advances, the process will become more automatic and will take less time. It will also become more available and affordable.

One of the main aims of virtual imaging is organ preservation in cases of locally advanced liver tumours. As for now, the impact of virtual imaging has rarely been analysed in the paediatric population. 3D reconstructions can allow for more aggressive surgical treatment when spatial relationships and crucial anatomical structures are depicted.

The conclusions of the reviewed articles are very enthusiastic, and the progress that has already been made in the field of computer-aided paediatric liver surgery is impressive. It is time to design studies appropriate for measuring the new technologies' effects on liver surgery outcomes.

The learning curves of those new technologies certainly have to be included in the study designs.

\section{DECLARATIONS}

\section{Authors' contributions}

Made substantial contributions to conception and design of the study and performed data analysis and interpretation: Kościuszko D, Anzelewicz S, Czauderna P

Participated in the discussion, prepared tables and shaped the overall structure: Niemirycz-Makurat A, Gołębiewski A, Kościuszko D

Prepared the final manuscript: Kościuszko D, Niemirycz-Makurat A, Anzelewicz S

\section{Availability of data and materials}

Not applicable.

\section{Financial support and sponsorship}

None.

\section{Conflicts of interest}

All authors declared that there are no conflicts of interest. 


\section{Ethical approval and consent to participate}

Not applicable.

\section{Consent for publication}

Not applicable.

\section{Copyright}

(C) The Author(s) 2021.

\section{REFERENCES}

1. Marescaux J, Clément JM, Tassetti V, et al. Virtual reality applied to hepatic surgery simulation: the next revolution. Ann Surg 1998;228:627-34. DOI PubMed PMC

2. Topol EJ. A decade of digital medicine innovation. Sci Transl Med 2019;11:eaaw7610. DOI PubMed

3. Kokudo N, Takemura N, Ito K, Mihara F. The history of liver surgery: Achievements over the past 50 years. Ann Gastroenterol Surg 2020;4:109-17. DOI PubMed PMC

4. Langridge B, Momin S, Coumbe B, Woin E, Griffin M, Butler P. Systematic review of the use of 3-dimensional printing in surgical teaching and assessment. J Surg Educ 2018;75:209-21. DOI PubMed

5. Witowski JS, Pędziwiatr M, Major P, Budzyński A. Cost-effective, personalized, 3D-printed liver model for preoperative planning before laparoscopic liver hemihepatectomy for colorectal cancer metastases. Int J Comput Assist Radiol Surg 2017;12:2047-54. DOI PubMed PMC

6. National Research Council (US) Committee on A Framework for Developing a New Taxonomy of Disease. Toward precision medicine: building a knowledge network for biomedical research and a new taxonomy of disease. Washington (DC): National Academies Press (US); 2011. DOI PubMed

7. Dong J, Yang S, Zeng J, et al. Precision in liver surgery. Semin Liver Dis 2013;33:189-203. DOI PubMed

8. Dong J, Huang Z. [Precise liver resection-new concept of liver surgery in 21st century]. Zhonghua Wai Ke Za Zhi 2009;47:1601-5. PubMed

9. JiaHong D, ZhiQiang H. To advocate precise hepatectomy and recreate the legend of Prometheus. Chinese J Dig Surg 2010;9:4-5.

10. Reardon MJ. See one, do one, teach one? J Thorac Cardiovasc Surg 2019;157:e45-6. DOI PubMed

11. Shakir HJ, Shallwani H, Levy EI. Editorial: see one, do one, teach one? Neurosurgery 2017;80:3-5. DOI PubMed

12. Moher D, Liberati A, Tetzlaff J, Altman DG; PRISMA Group. Preferred reporting items for systematic reviews and meta-analyses: the PRISMA statement. PLoS Med 2009;6:e1000097. DOI PubMed PMC

13. Han J, Kang HJ, Kim M, Kwon GH. Mapping the intellectual structure of research on surgery with mixed reality: bibliometric network analysis (2000-2019). J Biomed Inform 2020;109:103516. DOI PubMed

14. Quero G, Lapergola A, Soler L, et al. Virtual and augmented reality in oncologic liver surgery. Surg Oncol Clin N Am 2019;28:31-44. DOI PubMed

15. Agostini A, Borgheresi A, Floridi C, et al. The role of imaging in surgical planning for liver resection: what the radiologist need to know. Acta Biomed 2020;91:18-26. DOI PubMed PMC

16. Darge K, Papadopoulou F, Ntoulia A, et al. Safety of contrast-enhanced ultrasound in children for non-cardiac applications: a review by the Society for Pediatric Radiology (SPR) and the International Contrast Ultrasound Society (ICUS). Pediatr Radiol 2013;43:106373. DOI PubMed

17. Varich L. Ultrasound of pediatric liver masses. Ultrasound Clin 2010;5:137-52. DOI

18. Dietrich CF, Averkiou M, Nielsen MB, et al. How to perform Contrast-Enhanced Ultrasound (CEUS). Ultrasound Int Open 2018;4:E2-E15. DOI PubMed PMC

19. Claudon M, Dietrich CF, Choi BI, et al; World Federation for Ultrasound in Medicine, European Federation of Societies for Ultrasound. Guidelines and good clinical practice recommendations for Contrast Enhanced Ultrasound (CEUS) in the liver - update 2012: A WFUMB-EFSUMB initiative in cooperation with representatives of AFSUMB, AIUM, ASUM, FLAUS and ICUS. Ultrasound Med Biol 2013;39:187-210. DOI PubMed

20. Dietrich CF, Kratzer W, Strobe D, et al. Assessment of metastatic liver disease in patients with primary extrahepatic tumors by contrast-enhanced sonography versus CT and MRI. World J Gastroenterol 2006;12:1699-705. DOI PubMed PMC

21. Chiorean L, Caraiani C, Radzina M, Jedrzejczyk M, Schreiber-Dietrich D, Dietrich CF. Vascular phases in imaging and their role in focal liver lesions assessment. Clin Hemorheol Microcirc 2015;62:299-326. DOI PubMed

22. Seitz K, Strobel D, Bernatik T, et al. Contrast-Enhanced Ultrasound (CEUS) for the characterization of focal liver lesions - prospective comparison in clinical practice: CEUS vs. CT (DEGUM multicenter trial). Parts of this manuscript were presented at the Ultrasound Dreiländertreffen 2008, Davos. Ultraschall Med 2009;30:383-9. DOI PubMed

23. Sidhu PS, Cantisani V, Deganello A, et al. Role of Contrast-Enhanced Ultrasound (CEUS) in paediatric practice: an EFSUMB position statement. Ultraschall Med 2017;38:33-43. DOI PubMed

24. Thimm MA, Rhee D, Takemoto CM, et al. Diagnosis of congenital and acquired focal lesions in the neck, abdomen, and pelvis with contrast-enhanced ultrasound: a pictorial essay. Eur J Pediatr 2018;177:1459-70. DOI PubMed

25. McCollough CH, Primak AN, Braun N, Kofler J, Yu L, Christner J. Strategies for reducing radiation dose in CT. Radiol Clin North 
Am 2009;47:27-40. DOI PubMed PMC

26. You Y, Zhang M, Li K, et al. Feasibility of 3D US/CEUS-US/CEUS fusion imaging-based ablation planning in liver tumors: a retrospective study. Abdom Radiol (NY) 2021. DOI PubMed

27. Chung EM, Lattin GE Jr, Cube R, et al. From the archives of the AFIP: Pediatric liver masses: radiologic-pathologic correlation. Part 2. Malignant tumors. Radiographics 2011;31:483-507. DOI PubMed

28. McHugh K, Disini L. Commentary: for the children's sake, avoid non-contrast CT. Cancer Imaging 2011;11:16-8. DOI PubMed PMC

29. Siegel MJ. Pediatric liver imaging. Semin Liver Dis 2001;21:251-69. DOI PubMed

30. Semelka RC, Martin DR, Balci C, Lance T. Focal liver lesions: comparison of dual-phase CT and multisequence multiplanar MR imaging including dynamic gadolinium enhancement. J Magn Reson Imaging 2001;13:397-401. DOI PubMed

31. Oi H, Murakami T, Kim T, Matsushita M, Kishimoto H, Nakamura H. Dynamic MR imaging and early-phase helical CT for detecting small intrahepatic metastases of hepatocellular carcinoma. AJR Am J Roentgenol 1996;166:369-74. DOI PubMed

32. Yamashita Y, Mitsuzaki K, Yi T, et al. Small hepatocellular carcinoma in patients with chronic liver damage: prospective comparison of detection with dynamic MR imaging and helical CT of the whole liver. Radiology 1996;200:79-84. DOI PubMed

33. Siegel MJ, Chung EM, Conran RM. Pediatric liver: focal masses. Magn Reson Imaging Clin N Am 2008;16:437-52, v. DOI PubMed

34. Chavhan GB, Shelmerdine S, Jhaveri K, Babyn PS. Liver MR imaging in children: current concepts and technique. Radiographics 2016;36:1517-32. DOI PubMed

35. Jaspan ON, Fleysher R, Lipton ML. Compressed sensing MRI: a review of the clinical literature. Br J Radiol 2015;88:20150487. DOI PubMed PMC

36. Ditchfield M. 3T MRI in paediatrics: challenges and clinical applications. Eur J Radiol 2008;68:309-19. DOI PubMed

37. Schindera ST, Merkle EM, Dale BM, Delong DM, Nelson RC. Abdominal magnetic resonance imaging at $3.0 \mathrm{~T}$ what is the ultimate gain in signal-to-noise ratio? Acad Radiol 2006;13:1236-43. DOI PubMed

38. Seale MK, Catalano OA, Saini S, Hahn PF, Sahani DV. Hepatobiliary-specific MR contrast agents: role in imaging the liver and biliary tree. Radiographics 2009;29:1725-48. DOI PubMed

39. Cruite I, Schroeder M, Merkle EM, Sirlin CB. Gadoxetate disodium-enhanced MRI of the liver: part 2, protocol optimization and lesion appearance in the cirrhotic liver. AJR Am J Roentgenol 2010;195:29-41. DOI PubMed

40. Hamm B, Staks T, Mühler A, et al. Phase I clinical evaluation of Gd-EOB-DTPA as a hepatobiliary MR contrast agent: safety, pharmacokinetics, and MR imaging. Radiology 1995;195:785-92. DOI PubMed

41. Schooler GR, Hull NC, Lee EY. Hepatobiliary MRI contrast agents: pattern recognition approach to pediatric focal hepatic lesions. AJR Am J Roentgenol 2020;214:976-86. DOI PubMed

42. Chavhan GB, Mann E, Kamath BM, Babyn PS. Gadobenate-dimeglumine-enhanced magnetic resonance imaging for hepatic lesions in children. Pediatr Radiol 2014;44:1266-74. DOI PubMed

43. Marrone G, Maggiore G, Carollo V, Sonzogni A, Luca A. Biliary cystadenoma with bile duct communication depicted on liverspecific contrast agent-enhanced MRI in a child. Pediatr Radiol 2011;41:121-4. DOI PubMed

44. Meyers AB, Towbin AJ, Serai S, Geller JI, Podberesky DJ. Characterization of pediatric liver lesions with gadoxetate disodium. Pediatr Radiol 2011;41:1183-97. DOI PubMed

45. Joyner BL Jr, Levin TL, Goyal RK, Newman B. Focal nodular hyperplasia of the liver: a sequela of tumor therapy. Pediatr Radiol 2005;35:1234-9. DOI PubMed

46. Plumley DA, Grosfeld JL, Kopecky KK, Buckwalter KA, Vaughan W. The role of spiral (Helical) computerized tomography with three-dimensional reconstruction in pediatric solid tumors. J Pediatr Surg 1995;30:317-21. DOI PubMed

47. Fuchs J, Warmann SW, Szavay P, et al. Three-dimensional visualization and virtual simulation of resections in pediatric solid tumors. J Pediatr Surg 2005;40:364-70. DOI PubMed

48. Dong Q, Xu W, Jiang B, et al. Clinical applications of computerized tomography 3-D reconstruction imaging for diagnosis and surgery in children with large liver tumors or tumors at the hepatic hilum. Pediatr Surg Int 2007;23:1045-50. DOI PubMed

49. Fuchs J, Warmann SW, Sieverding L, et al. Impact of virtual imaging procedures on treatment strategies in children with hepatic vascular malformations. J Pediatr Gastroenterol Nutr 2010;50:67-73. DOI PubMed

50. Souzaki R, Ieiri S, Uemura M, et al. An augmented reality navigation system for pediatric oncologic surgery based on preoperative CT and MRI images. J Pediatr Surg 2013;48:2479-83. DOI PubMed

51. Souzaki R, Kinoshita Y, Ieiri S, et al. Three-dimensional liver model based on preoperative CT images as a tool to assist in surgical planning for hepatoblastoma in a child. Pediatr Surg Int 2015;31:593-6. DOI PubMed

52. Su L, Zhou XJ, Dong Q, et al. Application value of computer assisted surgery system in precision surgeries for pediatric complex liver tumors. Int J Clin Exp Med 2015;8:18406-12. PubMed PMC

53. Soejima Y, Taguchi T, Sugimoto M, et al. Three-dimensional printing and biotexture modeling for preoperative simulation in living donor liver transplantation for small infants. Liver Transpl 2016;22:1610-4. DOI PubMed

54. Su L, Dong Q, Zhang H, et al. Clinical application of a three-dimensional imaging technique in infants and young children with complex liver tumors. Pediatr Surg Int 2016;32:387-95. DOI PubMed

55. Warmann SW, Schenk A, Schaefer JF, et al. Computer-assisted surgery planning in children with complex liver tumors identifies variability of the classical Couinaud classification. J Pediatr Surg 2016;51:1801-6. DOI PubMed

56. Newe A, Becker L, Schenk A. Application and evaluation of interactive 3D PDF for presenting and sharing planning results for liver surgery in clinical routine. PLoS One 2014;9:e115697. DOI PubMed PMC

57. Zhang G, Zhou XJ, Zhu CZ, Dong Q, Su L. Usefulness of three-dimensional(3D) simulation software in hepatectomy for pediatric 
hepatoblastoma. Surg Oncol 2016;25:236-43. DOI PubMed

58. Janek J, Bician P, Kenderessy P, et al. [Experience with hepatoblastoma treatment in small children - the use of preoperative 3D virtual analysis MeVis for liver resections]. Rozhl Chir 2017;96:25-33. PubMed

59. Zhao J, Zhou XJ, Zhu CZ, et al. 3D simulation assisted resection of giant hepatic mesenchymal hamartoma in children. Comput Assist Surg (Abingdon) 2017;22:54-9. DOI PubMed

60. Wang P, Que W, Zhang M, et al. Application of 3-dimensional printing in pediatric living donor liver transplantation: a single-center experience. Liver Transpl 2019;25:831-40. DOI PubMed

61. Esaki T, Furukawa R. [Volume measurements of post-transplanted liver of pediatric recipients using workstations and deep learning]. Nihon Hoshasen Gijutsu Gakkai Zasshi 2020;76:1133-42. DOI PubMed

62. Ronneberger O, Fischer P, Brox T. U-Net: convolutional networks for biomedical image segmentation. Cham: Springer; 2015.

63. Ishii T, Fukumitsu K, Ogawa E, Okamoto T, Uemoto S. Living donor liver transplantation in situs inversus totalis with a patientspecific three-dimensional printed liver model. Pediatr Transplant 2020;24:e13675. DOI PubMed

64. Czauderna P, Lopez-Terrada D, Hiyama E, Häberle B, Malogolowkin MH, Meyers RL. Hepatoblastoma state of the art: pathology, genetics, risk stratification, and chemotherapy. Curr Opin Pediatr 2014;26:19-28. DOI PubMed

65. Meyers RL, Czauderna P, Otte JB. Surgical treatment of hepatoblastoma. Pediatr Blood Cancer 2012;59:800-8. DOI PubMed

66. Murawski M, Łosin M, Gołębiewski A, et al. Laparoscopic resection of liver tumors in children. J Pediatr Surg 2021;56:420-3. DOI PubMed

67. Cai X. Laparoscopic liver resection: the current status and the future. Hepatobiliary Surg Nutr 2018;7:98-104. DOI PubMed PMC

68. Marescaux J, Rubino F, Arenas M, Mutter D, Soler L. Augmented-reality-assisted laparoscopic adrenalectomy. JAMA 2004;292:22145. DOI PubMed

69. Haouchine N, Dequidt J, Berger M-O, Cotin S. Deformation-based augmented reality for hepatic surgery. Stud Health Technol Inform 2013;184:182-8. PubMed

70. Abdalla EK, Barnett CC, Doherty D, Curley SA, Vauthey JN. Extended hepatectomy in patients with hepatobiliary malignancies with and without preoperative portal vein embolization. Arch Surg 2002;137:675-80; discussion 680. DOI PubMed

71. Vauthey JN, Chaoui A, Do KA, et al. Standardized measurement of the future liver remnant prior to extended liver resection: methodology and clinical associations. Surgery 2000;127:512-9. DOI PubMed

72. Shoup M. Volumetric analysis predicts hepatic dysfunction in patients undergoing major liver resection. J Gastrointest Surg 2003;7:325-30. DOI PubMed

73. Ripley B, Levin D, Kelil T, et al. 3D printing from MRI Data: Harnessing strengths and minimizing weaknesses. J Magn Reson Imaging 2017;45:635-45. DOI PubMed

74. van der Vorst JR, van Dam RM, van Stiphout RS, et al. Virtual liver resection and volumetric analysis of the future liver remnant using open source image processing software. World J Surg 2010;34:2426-33. DOI PubMed PMC

75. Dello SA, Stoot JH, van Stiphout RS, et al. Prospective volumetric assessment of the liver on a personal computer by nonradiologists prior to partial hepatectomy. World J Surg 2011;35:386-92. DOI PubMed PMC

76. Dello SA, van Dam RM, Slangen JJ, et al. Liver volumetry plug and play: do it yourself with ImageJ. World J Surg 2007;31:2215-21. DOI PubMed PMC

77. Lodewick TM, Arnoldussen CW, Lahaye MJ, et al. Fast and accurate liver volumetry prior to hepatectomy. HPB (Oxford) 2016;18:764-72. DOI PubMed PMC

78. Ibtehaz N, Rahman MS. MultiResUNet : Rethinking the U-Net architecture for multimodal biomedical image segmentation. Neural Netw 2020;121:74-87. DOI PubMed

79. Heimann T, van Ginneken B, Styner MA, et al. Comparison and evaluation of methods for liver segmentation from CT datasets. IEEE Trans Med Imaging 2009;28:1251-65. DOI PubMed

80. Tian Y, Xue F, Lambo R, et al. Fully-automated functional region annotation of liver via a 2.5D class-aware deep neural network with spatial adaptation. Comput Methods Programs Biomed 2021;200:105818. DOI PubMed

81. Winkel DJ, Weikert TJ, Breit HC, et al. Validation of a fully automated liver segmentation algorithm using multi-scale deep reinforcement learning and comparison versus manual segmentation. Eur J Radiol 2020;126:108918. DOI PubMed

82. Witowski JS, Coles-Black J, Zuzak TZ, et al. 3D Printing in liver surgery: a systematic review. Telemed J E Health 2017;23:943-7. DOI PubMed

83. Tack P, Victor J, Gemmel P, Annemans L. 3D-printing techniques in a medical setting: a systematic literature review. Biomed Eng Online 2016;15:115. DOI PubMed PMC

84. Chen H, He Y, Jia W. Precise hepatectomy in the intelligent digital era. Int J Biol Sci 2020;16:365-73. DOI PubMed PMC

85. Gotra A, Sivakumaran L, Chartrand G, et al. Liver segmentation: indications, techniques and future directions. Insights Imaging 2017;8:377-92. DOI PubMed PMC

86. Saito Y, Sugimoto M, Imura S, et al. Intraoperative 3D hologram support with mixed reality techniques in liver surgery. Ann Surg 2020;271:e4-7. DOI PubMed

87. Elshafei M, Binder J, Baecker J, et al. Comparison of cinematic rendering and computed tomography for speed and comprehension of surgical anatomy. JAMA Surg 2019;154:738-44. DOI PubMed PMC

88. Binder JS, Scholz M, Ellmann S, et al. Cinematic rendering in anatomy: a crossover study comparing a novel 3D reconstruction technique to conventional computed tomography. Anat Sci Educ 2021;14:22-31. DOI PubMed

89. Chu LC, Rowe SP, Fishman EK. Cinematic rendering of focal liver masses. Diagn Interv Imaging 2019;100:467-76. DOI PubMed 